\title{
Functional role of increased acetylated tubulin in porcine oocyte microtubule structure, meiotic maturation and embryogenesis
}

Junming Sun ( $\nabla$ sjm990205@163.com )

Guangxi Medical University https://orcid.org/0000-0001-9242-721X

Kuiqing Cui

Guangxi University

Zhipeng Li

Guangxi University

Bangjun Gao

Guangxi University

Jianrong Jiang

Guangxi University

Qingyou Liu

Guangxi University

Ben Huang

Guangxi University

Denshun Shi

Guangxi University

Research article

Keywords: microtubule, IVF, a-tubulin, acetylation, mitochondria.

Posted Date: December 17th, 2019

DOI: https://doi.org/10.21203/rs.2.19001/v1

License: (c) (i) This work is licensed under a Creative Commons Attribution 4.0 International License.

Read Full License 


\section{Abstract}

Acetylated microtubule improves porcine oocyte microtubule structure, meiotic maturation and subsequent embryonic development. HDAC6 can specifically deacetylate a-tubulin in assembled microtubules, increased acetylated microtubule treatment with tubacin, a HDAC6-selective inhibitor, is beneficial for porcine oocytes maturation and early embryogenesis. Here it is shown that a-tubulin acetylation gradually decreased from MI to IVF pronuclear stage. The increased acetylation of a-tubulin significantly reduced the abnormal rate of microtubules, furthermore, the proportion of mitochondria in the vicinity of IVF nucleus was significantly enhanced in MI and MII stages. The expression levels of microtubule assembly genes ( TUBA1A , a TAT1 and MAP2 ) significantly up-regulated in MI and MII stages. In addition, the oocytes with high acetylation level of a-tubulin significantly improved maturation, syngamy and IVF blastocyst formation compared with the control oocytes. In present study, these indicate functional role of increased acetylated a-tubulin advances normal spindle formation and mitochondrial concentration, moreover, improves porcine maturation, syngamy and preimplantation embryo development.

\section{Background}

Microtubules (MTs) are required for the cascade of events including the spindle assembly, mitochondria distribution, meiotic maturation, syngamy and early embryo development(1-4). The inhibition of microtubule assembly has adverse effects on these important events in different species $(1,5-7)$. Abnormal microtubule is caused by aging(8), in vitro maturation(9), chemical exposures(10) and mutations(11). Some drugs could hinder microtubule assembly (colchicine(12) and nocodazole(13)) or prevent depolymerization (taxol(14)) during oocytes meiotic maturation. These data suggest that the disruption of dynamic balance may result in abnormalities of microtubules.

Acetylated microtubule is a reversible post-translational modifications (PTMs) which catalyzes the acetylation of Lys-40 sites on the inner surface of microtubules(15). Although it is not clear whether the stability of microtubules is related to the acetylation of microtubules $(16,17)$, acetylated tubulin appears more stable treated by colchicine $(12)$ and nocodazole $(13,18)$. With TSA and Tubacin treatment or catalytically inactive HDAC6, increased acetylated tubulin enhances the chemotactic motility of these cells $(19,20)$. The acetylated a-tubulin of mouse is predominantly at the spindle of MII and first fertilization cleavage(21). HDAC6 knockout in mice increased microtubules acetylation without affecting the growth and development(22). It is still unclear how the functional roles of increased acetylated alters maturation, fertilization, and early embryonic development.

Histone deacetylase 6 (HDAC6) removed the a-K40 acetylation (19), and localized to the cytoplasm and operated on the free tubulin dimer. Tubacin, a selective and reversible HDAC6 inhibitor, could increase atubulin acetylation to make microtubules dynamic and stable $(20,23)$. aTAT1 catalyzes the a-K40 acetylation (24) and is homology to histone acetyltransferases(25). The recruitment of microtubuleassociated proteins (MAPs) into acetylated tubulin can lead the change in dynamic balance (26-28). 
However, it has not been addressed whether increased a-tubulin acetylation causes the changes of aTAT1 and MAPs in oocyte maturation and fertilization.

Our understanding of increased a-tubulin acetylation should keep stable after microtubules assembly, however, not disrupt dynamic balance of microtubules structure to advance the maturation, fertilization and development. We explored the functional roles of increased a-tubulin acetylation in spindle formation, mitochondrial distribution, PN syngamy, embryo development, and the transcription of atubulin acetyltransferase and microtubule-associated proteins in porcine oocyte maturation and fertilization.

\section{Results}

\section{Effect of drug treatment on the a-tubulin acetylation}

As indicated by mean pixel intensity after immunofluorescence staining (Fig. 1), the dynamic pattern of atubulin acetylation gradually decreased from MI to MII to PN syngamy. The intensity of a-tubulin acetylation treatment with tubacin were stronger than control in $\mathrm{MI}$ and $\mathrm{MII}(P<0.05)$, but they were not different in PN syngamy ( $P>0.05)$.

\section{Effect of increased a-tubulin acetylation on spindle morphology}

GV oocytes displayed a large number of a-tubulin in the cortex (Fig. 2A). The normal spindle was barrel shaped or slightly pointed (Fig. 2B,D,E,H), and the abnormal spindle was disorganized, asymmetrical, round or elongated (Fig. 2C,F,G,I). With increased a-tubulin acetylation, the normal spindle was higher than the control in $\mathrm{Ml}$ and syngamy ( $87.73 \%$ vs. $70.93 \%$ and $22.22 \%$ vs. $11.94 \%, P<0.05$; Table 2$)$, and the abnormal spindles were lower than the control in MI and MII (4.94 \% vs. $20.25 \%$ and $17.26 \%$ vs. $35.65 \%, P<0.05$; Table 2). These suggest that the increased a-tubulin acetylation have a critical effect to improve microtubules after meiotic apparatus assembly.

\section{Effect of increased a-tubulin acetylation on mitochondrial distribution and fertilization process}

The mitochondria aggregation in the vicinity of treated nucleus was more obvious than the control (47.02 $\%$ vs. $25.59 \%, P<0.05$; Table 3$)$. After in vitro fertilization, The mitochondria aggregation was associated with the pronucleus (Fig. 3B) or syngamy chromosome (Fig. 3C). With increased a-tubulin acetylation, the syngamy proportion was higher than the control (16.04 vs. $6.15, \mathrm{P}<0.05$; Table 4$)$. These indicated that increased a-tubulin acetylation can promote mitochondria concentration and pronucleus formation. 
The rates of maturation and blastocyst on the treated groups were higher than the control $(72.90 \pm 5.13 \%$ vs. $64.06 \pm 2.87 \% ; 13.28 \pm 1.79 \%$ vs. $9.80 \pm 1.93 \%$; $P<0.05$; Table 5). However, there were not different in 2 -cells and total number of blastocyst cells between two groups $(P>0.05)$. These showed that the functional effects of increased a-tubulin acetylation can improve in vitro maturation and fertilization development.

\section{Effect of increased a-tubulin acetylation on the transcription of related genes}

From $\mathrm{Ml}$ to $\mathrm{MII}$, the expression of $a T A T 1, M A P 2, H S P 90$ and Katanin was significantly up-regulated $(P<0.05)$, but the expression of TUBA1A was significantly down-regulated $(P<0.05)$ (Fig. 5$)$. At the $\mathrm{MI}$ stage, the TUBA1A transcription in the treatment had significantly higher levels than the control $(P<0.05)$ (Fig. 5A). The aTAT1 and MAP2 patterns of the MII oocytes in the treatment had significantly higher expression than the control $(P<0.05)$ (Fig.5B,5C). The results support a molecular mechanism of increased a-tubulin acetylation for recruiting MAPs to improve the configuration of microtubule dynamics. The above results indicated that increased a-tubulin acetylation promoted the binding of tubulin to MAPs and motor proteins, which improved microtubule configuration, maturation and fertilization.

\section{Discussion}

Tubulin acetylation could affect microtubule associated proteins recruitment to microtubules and alter microtubule assemble(29). The study investigated the dynamic pattern of a-tubulin acetylation gradually decreased from MI to MII to PN syngamy. The intensity of a-tubulin acetylation treatment with tubacin was significantly higher in MI and MII (Fig. 1), but they were not different in PN syngamy. Abnormal microtubule acetylation of GV oocytes influenced spindle structure, which lead to abnormal maturation and failure of chromosome division(30). Thus, tubacin increased porcine a-tubulin acetylation from $\mathrm{MI}$ to MII stages. With increased a-tubulin acetylation, the normal rates of spindle formation were significantly higher than the control in $\mathrm{Ml}$ and syngamy, and the abnormalities of $\mathrm{MI}$ and $\mathrm{MII}$ spindles were significantly lower (Table 2). The oocyte development required microtubule assembly and chromosome association. Post-translational modifications promoted microtubule associated proteins binding to the microtubule, which affected microtubule assemble and dynamic stability to perform different physiological functions. Some drugs could hinder microtubule assembly (colchicine(12) and nocodazole $(13,18)$ ) or prevent depolymerization (taxol(14)) during oocytes meiotic maturation, which significantly inhibit spindle morphology in vitro maturation and fertilization. These data supported increased porcine a-tubulin acetylation is critical to microtubules dynamic stabilization in meiotic maturation and fertilization.

This study showed the proportion of mitochondria aggregation in the vicinity of treated nucleus was significantly higher (Table 3). The mitochondrion of fertilized egg was close to the pronucleus (Fig. 3B), or associated with syngamy chromosome (Fig. 3C). Mitochondria represented the areas of ATP 
concentration that contributed to embryonic development. Microtubules were thought to promote mitochondrial transport and concentration in neuron cell (31). Microtubules of porcine oocytes could regulate active mitochondrial distribution to improve the embryo development (32). The abnormal mitochondrion would greatly affect early embryo development, reduce the post-implantation development of the fetus and even lead to diseases in adulthood (33). Thus, it was conducted orcein stain to analyze changes in fertilization process. Increased a-tubulin acetylation embryos showed higher proportion of the syngamy than the control embryos (Table 4). Microtubule is the key factor affecting the fusion of male and female pronucleus in mouse fertilized eggs(1). The functional effects of increased a-tubulin acetylation can promote mitochondria concentration distribution and pronucleus formation. It was required to test maturation efficiency and embryo development. The rates of maturation and blastocyst were significantly enhanced (Table 5). The cumulative data suggest that increased a-tubulin acetylation have a critical effect in oocytes maturation and fertilization.

Post-translational modifications of the microtubules were in the C-terminal tails or on the outer surface of microtubules. The modifications of a-tubulin were closely linked to microtubule-associated proteins, and specifc modifications would change the interaction between a-tubulin and microtubule-associated proteins. It was determined to measure the relative level of $a T A T 1$ and MAP2 in oocytes maturation. From $\mathrm{MI}$ to MII, the expression of aTAT1 and MAP2 significantly rose (Fig. 5). The aTAT1 and MAP2 patterns of the treated MII oocytes were higher than the control (Fig.5B, C). aTAT1 is a member of GCN 5 superfamily of acetyltransferase, and is still not clear to catalyze acetylation. aTAT1 may be the only enzyme to catalyze acetylation in K40 site, and tubulin acetylation plays a key role in cytoplasmic structure (24). MAP2 can bind microtubules to promote dynamic stability and extension. Phosphorylation and dephosphorylation can reversibly regulate the binding of MAP2 and microtubule. MAP2 dephosphorylation can promote the dynamic stability of microtubules $(34,35)$. Few studies have focused on the relationship between the MAP2 and maturation. The study shows that increased levels of a-tubulin acetylation can promote the expression of $M A P 2$, which is conducive to microtubule stability. These results support a molecular mechanism of increased a-tubulin acetylation for recruiting aTAT1 and MAP2 to improve the configuration of microtubule dynamics.

\section{Conclusions}

The hyperacetylation of a-tubulin significantly reduced the abnormal rate of microtubules, furthermore, the proportion of mitochondria in the vicinity of IVF nucleus was significantly enhanced in $\mathrm{MI}$ and $\mathrm{MII}$ stages. The expression levels of microtubule assembly genes (TUBA1A, aTAT1 and MAP2) significantly up-regulated in $\mathrm{MI}$ and MII stages. In addition, the oocytes with high acetylation level of a-tubulin significantly improved maturation, syngamy and IVF blastocyst formation compared with the control oocytes. These suggest that functional role of increased acetylated a-tubulin promotes normal spindle formation and mitochondrial concentration, moreover, improves porcine maturation, syngamy and preimplantation embryo development. The present work provides evidence supporting a functional role of acetylated microtubule in porcine oocyte microtubule structure and that specific stimulatory actions of meiotic maturation and early embryogenesis are observed. 


\section{Materials And Methods}

Our chemicals and reagents were from the Sigma-Aldrich. All experiments were performed according to the guidelines of Guangxi University.

\section{In vitro maturation}

Oocyte maturation process and formula follow our previous experiments(36). Cumulus oocyte complexes (COCs) were cultured in the maturation medium containing $15 \mathrm{IU} / \mathrm{mL}$ eCG and $10 \mathrm{lU} / \mathrm{mL} \mathrm{hCG}$ for 20-22h, and transferred to free hormone maturation medium for the remaining 20-22h. Maturation medium was supplemented with tubacin (20mM stock in DMSO, $2 \mu \mathrm{M}$ final; selleck Chemicals) during MI (28h) or MII (44h). Oocytes treated with tubacin were use for the experiments of in vitro fertilization or fluorescence labeling.

\section{Production of IVF embryos}

Fertilization process and formula follow our previous experiments(36). The matured cumulus was gently blown and digested. Oocytes with polar body and uniform cytoplasm were coincubated with semen of appropriate concentration for $4 \mathrm{~h}$. The presumptive zygotes were selected for subsequent experiments or cultured in PZM-3. The rates of cleavage and blastocyst were recorded at $24 \mathrm{~h}$ and $168 \mathrm{~h}$. The total number of blastocysts was recorded after staining.

\section{Confocal microscopy}

The Immunofluorescence procedures were performed according to our previous study(36). Samples were incubated with a-tubulin antibody (1:100; Santa cruz) and a-tubulin acetylation antibody (1:100; Proteintech) primary antibodies, and was labeled with fluorescein isothiocyanate co-njugated (1:200; Sigma) secondary antibody. Live oocytes or embryos was incubated in PZM-3 containing Mito-tracker Green (Molecular Probes). They were detected by a confocal microscope (Cofocal microscope, Leica, Inc.). The brightest area of every sample was automatically quantified and recorded. The fluorescence intensity represents the acetylation level used for statistical analysis.

\section{Evaluation of in vitro fertilization}

After IVF 22-24 h (starting from culture), After IVF 22-24 h (starting from culture), presumptive zygotes were transferred into PBS on the slide. The four corners of the droplet was dotted with Vaseline. The 
cover glass was gently pressed down to keep oocytes intact on vaseline. About $100 \mu \mathrm{L}$ of fixing liquid (3:1 ethanol:acetic acid) was carefully pushed into the space between the cover glass and the slide. According to the above method, $1 \%(\mathrm{w} / \mathrm{v})$ orcein was gently pushed to cover fixed oocyte. The changes of fertilization pronucleus were observed and recorded under inverted microscope at 200x.

\section{Reverse transcription and QRT-PCR}

Reverse transcription and QRT-PCR procedures were performed according to our previous study (36). These primers (table 1) were synthesized from Sangon Biotech (Shanghai, China). Five embryos was collected into the lysis solution, and messenger RNA was reverse transcribed. The expression of different genes was automatically acquired and recorded. Each gene in different sample was repeated three times, and detected compared with the expression of the endogenous control 18s. The expression levels of the genes were calculated by $2^{-} \Delta \Delta \mathrm{CT}$ calculation method.

\section{Statistics}

Results are represented as mean SEM. Statistical analysis was proceeded by one-way analysis of variance and tested by Duncan's multiple comparison. Only $p<0.05$ represented significant difference.

\section{Declarations}

Our experiment was approved by the animal ethics committee of Guangxi University. Each of our authors agreed to publish. Thank all the authors for their hard work. Thanks to Professor Deshun Shi, Professor Qingyou Liu, Professor Ben Huang and Professor Kuiqing Cui for guiding the experiment. Thanks to Dr. Zhipeng Li and Dr. Bangjun Gao for qRT-PCR experiment, immunofluorescence experiment and data collection. Thanks to Ms. Jianrong Jiang for her interest in the experiment. We have no conflict of interest. This study was supported by grants from the China National High Technology Research and Development Program (863) Project (2011AA100607), National Natural Science Foundation Project (31260552 and 31401267), Guangxi Natural Science Foundation (Grant No. 2014GXNSFCB118003) and Guangxi Medical University Youth Science Foundation Project (GXMUYSF201829).

\section{References}

1. Schatten G, Simerly C, Schatten H. Microtubule configurations during fertilization, mitosis, and early development in the mouse and the requirement for egg microtubule-mediated motility during mammalian fertilization. Proc Natl Acad Sci U S A. [; Research Support, U.S. Gov't, Non-P.H.S.; Research Support, U.S. Gov't, P.H.S.]. 1985 1985;82(12):4152-6. 
2. Bavister BD, Squirrell JM. Mitochondrial distribution and function in oocytes and early embryos. Human reproduction (Oxford, England). [; Research Support, U.S. Gov't, Non-P.H.S.; Research Support, U.S. Gov't, P.H.S.; Review]. 2000 2000;15 Suppl 2:189-98.

3. Tremoleda JL, van Haeften T, Stout TAE, Colenbrander B, Bevers MM. Cytoskeleton and Chromatin Reorganization in Horse Oocytes Following Intracytoplasmic Sperm Injection: Patterns Associated with Normal and Defective Fertilization. Biology of Reproduction. 2003;69(1):186-94.

4. Schatten H, Sun Q-Y. Posttranslationally Modified Tubulins and Other Cytoskeletal Proteins: Their Role in Gametogenesis, Oocyte Maturation, Fertilization and Pre-implantation Embryo Development. 2014;759:57-87.

5. Kim NH, Cho SK, Choi SH, Kim EY, Park SP, Lim JH. The distribution and requirements of microtubules and microfilaments in bovine oocytes during in vitro maturation. Zygote. [Article]. 2000 Feb;8(1):25-32.

6. Kim NH, Funahashi H, Prather RS, Schatten G, Day BN. Microtubule and microfilament dynamics in porcine oocytes during meiotic maturation. Molecular Reproduction and Development. [Article]. 1996 Feb;43(2):248-55.

7. Kim NH, Chung KS, Day BN. The distribution and requirements of microtubules and microfilaments during fertilization and parthenogenesis in pig oocytes. Journal of reproduction and fertility. [Article]. 1997 Sep;111(1):143-9.

8. Steuerwald N, Cohen J, Herrera RJ, Sandalinas M, Brenner CA. Association between spindle assembly checkpoint expression and maternal age in human oocytes. Molecular Human Reproduction. [Article]. 2001 Jan;7(1):49-55.

9. Sanfins A, Lee GY, Plancha CE, Overstrom EW, Albertini DF. Distinctions in Meiotic Spindle Structure and Assembly During In Vitro and In Vivo Maturation of Mouse Oocytes1. Biology of Reproduction. 2003;69(6):2059-67.

10. Yin H, Cukurcam S, Betzendahl I, Adler ID, Eichenlaub-Ritter U. Trichlorfon exposure, spindle aberrations and nondisjunction in mammalian oocytes. Chromosoma. [Article]. 1998 Dec;107(67):514-22.

11. Libby BJ, De La Fuente R, O'Brien MJ, Wigglesworth K, Cobb J, Inselman A, et al. The Mouse Meiotic Mutation mei1 Disrupts Chromosome Synapsis with Sexually Dimorphic Consequences for Meiotic Progression. Developmental Biology. 2002;242(2):174-87.

12. De Brabander MJ VdVR, Aerts FE, Borgers M, Janssen PA. The effects of methyl (5-(2thienylcarbonyl)-1H-benzimidazol-2-yl) carbamate, (R 17934; NSC 238159), a new synthetic antitumoral drug interfering with microtubules, on mammalian cells cultured in vitro. CANCER RES. [Comparative Study;]. 1976 1976;36(3):905-16.

13. Piperno G, LeDizet $M$, Chang XJ. Microtubules containing acetylated alpha-tubulin in mammalian cells in culture. The Journal of Cell Biology. [; Research Support, Non-U.S. Gov't; Research Support, U.S. Gov't, P.H.S.]. 1987 1987;104(2):289-302. 
14. Kim NH, Chung HM, Cha KY, Chung KS. Microtubule and microfilament organization in maturing human oocytes. Human Reproduction. [Article]. 1998 Aug;13(8):2217-22.

15. Alushin Gregory M, Lander Gabriel C, Kellogg Elizabeth H, Zhang R, Baker D, Nogales E. HighResolution Microtubule Structures Reveal the Structural Transitions in a $\beta$-Tubulin upon GTP Hydrolysis. Cell. 2014;157(5):1117-29.

16. Maruta H, Greer K, Rosenbaum JL. The acetylation of alpha-tubulin and its relationship to the assembly and disassembly of microtubules. The Journal of Cell Biology. [; Research Support, U.S. Gov't, P.H.S.]. 1986 1986;103(2):571-9.

17. Li L, Yang X-J. Tubulin acetylation: responsible enzymes, biological functions and human diseases. Cellular and Molecular Life Sciences. 2015;72(22):4237-55.

18. LeDizet M, Piperno G. Cytoplasmic microtubules containing acetylated alpha-tubulin in Chlamydomonas reinhardtii: spatial arrangement and properties. The Journal of Cell Biology. [; Research Support, Non-U.S. Gov't; Research Support, U.S. Gov't, P.H.S.]. 1986 1986;103(1):13-22.

19. Hubbert C, Guardiola A, Shao R, Kawaguchi Y, Ito A, Nixon A, et al. HDAC6 is a microtubuleassociated deacetylase. Nature. [Article]. 2002 May;417(6887):455-8.

20. Zilberman Y, Ballestrem C, Carramusa L, Mazitschek R, Khochbin S, Bershadsky A. Regulation of microtubule dynamics by inhibition of the tubulin deacetylase HDAC6. Journal of Cell Science. 2009;122(19):3531-41.

21. Schatten G, Simerly C, Asai DJ, Szoke E, Cooke P, Schatten H. Acetylated alpha-tubulin in microtubules during mouse fertilization and early development. Developmental Biology. [; Research Support, U.S. Gov't, Non-P.H.S.; Research Support, U.S. Gov't, P.H.S.]. 1988 1988;130(1):74-86.

22. Zhang Y, Kwon S, Yamaguchi T, Cubizolles F, Rousseaux S, Kneissel M, et al. Mice lacking histone deacetylase 6 have hyperacetylated tubulin but are viable and develop normally. Molecular and Cellular Biology. [Article]. 2008 Mar;28(5):1688-701.

23. Haggarty SJ, Koeller KM, Wong JC, Grozinger CM, Schreiber SL. Domain-selective small-molecule inhibitor of histone deacetylase 6 (HDAC6)-mediated tubulin deacetylation. Proceedings of the National Academy of Sciences. 2003;100(8):4389-94.

24. Shida T, Cueva JG, Xu ZJ, Goodman MB, Nachury MV. The major alpha-tubulin K40 acetyltransferase alpha TAT1 promotes rapid ciliogenesis and efficient mechanosensation. Proc Natl Acad Sci U S A. [Article]. 2010 Dec;107(50):21517-22.

25. Friedmann DR, Aguilar A, Fan J, Nachury MV, Marmorstein R. Structure of the -tubulin acetyltransferase, TAT1, and implications for tubulin-specific acetylation. Proceedings of the National Academy of Sciences. 2012;109(48):19655-60.

26. Dompierre JP, Godin JD, Charrin BC, Cordelieres FP, King SJ, Humbert S, et al. Histone deacetylase 6 inhibition compensates for the transport deficit in Huntington's disease by increasing tubulin acetylation. J Neurosci. [Article]. 2007 Mar;27(13):3571-83.

27. Reed NA, Cai DW, Blasius TL, Jih GT, Meyhofer E, Gaertig J, et al. Microtubule acetylation promotes kinesin-1 binding and transport. Current Biology. [Article]. 2006 Nov;16(21):2166-72. 
28. Sudo H, Baas PW. Acetylation of Microtubules Influences Their Sensitivity to Severing by Katanin in Neurons and Fibroblasts. J Neurosci. [Article]. 2010 May;30(21):7215-26.

29. Magiera MM, Janke C. Post-translational modifications of tubulin. Current Biology. 2014;24(9):R351R4.

30. Wang $\mathrm{H}-\mathrm{H}$, Zhang $\mathrm{Y}$, Tang F, Pan M-H, Wan X, Li X-H, et al. Rab23/Kif17 regulate meiotic progression in oocytes by modulating tubulin acetylation and actin dynamics. Development. 2019;146(3):dev171280.

31. Hirokawa N, Niwa S, Tanaka Y. Molecular Motors in Neurons: Transport Mechanisms and Roles in Brain Function, Development, and Disease. Neuron. 2010;68(4):610-38.

32. Sun QY, Wu GM, Lai L, Park KW, Cabot R, Cheong HT, et al. Translocation of active mitochondria during pig oocyte maturation, fertilization and early embryo development in vitro. Reproduction. [Article]. 2001 Jul;122(1):155-63.

33. Harvey AJ. Mitochondria in early development: linking the microenvironment, metabolism and the epigenome. Reproduction. 2019;157(5):R159-R79.

34. Kaźmierczak-Barańska J, Pęczek Ł, Przygodzka P, Cieślak MJ. Downregulation of striatin leads to hyperphosphorylation of MAP2, induces depolymerization of microtubules and inhibits proliferation of HEK293T cells. FEBS Lett. 2015;589(2):222-30.

35. Murthy AS FM. Microtubule assembly using the microtubule-associated protein MAP-2 prepared in defined states of phosphorylation with protein kinase and phosphatase. Eur J Biochem 1983;137(null):37-46.

36. Sun JM, Cui KQ, Li ZP, Lu XR, Xu ZF, Liu QY, et al. Suberoylanilide hydroxamic acid, a novel histone deacetylase inhibitor, improves the development and acetylation level of miniature porcine handmade cloning embryos. Reproduction in Domestic Animals. 2017;52(5):763-74.

\section{Tables}

Table 1. Primer Sequences, and Expected Product Sizes for QRT-PCR Analysis 


\begin{tabular}{|c|c|c|c|}
\hline $\begin{array}{l}\text { Target } \\
\text { Genes }\end{array}$ & Primers Sequences (5’to 3’) & $\begin{array}{c}\text { GenBank Accession } \\
\text { Number }\end{array}$ & $\begin{array}{l}\text { Product Size } \\
\text { (bp) }\end{array}$ \\
\hline \multirow[t]{2}{*}{$18 s$} & F: GATGGGCGGCGGAAAATTG & NR_046261 & 107 \\
\hline & R: TCCTCAACACCACATGAGCA & & \\
\hline \multirow[t]{4}{*}{ TUBA1A } & $\mathrm{F}:$ & NM_001315710 & 163 \\
\hline & СTCCCTGCTGATGGAACGTCT & & \\
\hline & $\mathrm{R}:$ & & \\
\hline & TACCATGAAGGCACAATCAGAG & & \\
\hline \multirow[t]{4}{*}{$\alpha T A T 1$} & F: & XM_005665740 & 170 \\
\hline & CGCCTTCTGCTCGCTACTGACC & & \\
\hline & $\mathrm{R}:$ & & \\
\hline & GTTTCCTGCTCCCCCTGCTTCA & & \\
\hline \multirow[t]{2}{*}{ MAP2 } & F: ACAGAAGACAGAACCAAGCC & XM_013984450 & 131 \\
\hline & R: CAACTAAACCCCACTCGTCC & & \\
\hline \multirow[t]{4}{*}{ HSP9O } & F: & NM_213973 & 103 \\
\hline & CAAACACAACGATGACGAGCAG & & \\
\hline & $\mathrm{R}:$ & & \\
\hline & AACCTTTGTTCCACGACCCAT & & \\
\hline \multirow[t]{2}{*}{ Katanin } & F: CGGAACGATTGCTGAAACCT & XM_013991829 & 155 \\
\hline & R: TTTGACTAACTGCTTGGCTG & & \\
\hline
\end{tabular}

Table 2. Effects of increased $\alpha$-tubulin acetylation on the microtubules configuration

\begin{tabular}{|c|c|c|c|c|c|c|c|}
\hline $\mathrm{s}$ & $\begin{array}{c}\text { Normal MI } \\
(\%)\end{array}$ & $\begin{array}{c}\text { Abnormal } \\
\text { MI } \\
\text { (\%) }\end{array}$ & $\begin{array}{c}\text { Others } \\
(\%)\end{array}$ & $\begin{array}{c}\text { Normal } \\
\text { MII } \\
(\%)\end{array}$ & $\begin{array}{c}\text { Abnormal } \\
\text { MII } \\
\text { (\%) }\end{array}$ & $\begin{array}{c}\text { Others } \\
(\%)\end{array}$ & $\begin{array}{c}\text { IVF tubulin } \\
\text { (\%) }\end{array}$ \\
\hline 1 & $37(70.93)^{b}$ & $12(20.25)^{\mathrm{a}}$ & $5(8.82)$ & $28(62.26)$ & $16(35.65)^{\mathrm{a}}$ & $1(2.22)$ & $5(11.94)^{b}$ \\
\hline & $36(87.73)^{\mathrm{a}}$ & $2(4.94)^{b}$ & $3(7.14)$ & $32(70.24)$ & $8(17.26)^{b}$ & $6(12.80)$ & $8(22.22)^{\mathrm{a}}$ \\
\hline
\end{tabular}


a, b Values with different superscripts are significantly different $(\mathrm{P}<0.05)$.

Table 3. Effects of increased $\alpha$-tubulin acetylation on the mitochondrial distribution

\begin{tabular}{ccccc}
\hline Groups & Oocytes & PN syngamy focus(\%) & PN syngamy diffusion (\%) & Others(\%) \\
\hline Control & 126 & $28(25.59)^{\mathrm{b}}$ & $83(65.68)^{\mathrm{a}}$ & $15(8.73)$ \\
Treat & 123 & $51(47.02)^{\mathrm{a}}$ & $58(47.01)^{\mathrm{b}}$ & $14(5.97)$ \\
\hline
\end{tabular}

a, b Values with different superscripts are significantly different $(\mathrm{P}<0.05)$.

Table 4. Effects of increased $\alpha$-tubulin acetylation on the IVF process

\begin{tabular}{cccccc}
\hline Groups & Oocytes & Syngamy(\%) & Unfertilized(\%) & Polysperm(\%) & Others(\%) \\
\hline Control & 227 & $15(6.15)^{\mathrm{b}}$ & $55(25.34)$ & $120(48.95)$ & $37(19.56)$ \\
Treat & 314 & $52(16.04)^{\mathrm{a}}$ & $67(21.32)$ & $157(48.73)$ & $38(13.91)$ \\
\hline
\end{tabular}

a, b Values with different superscripts are significantly different $(\mathrm{P}<0.05)$.

Table 5. Effects of increased $\alpha$-tubulin acetylation on the maturation and IVF embryos

\begin{tabular}{cccccc}
\hline Groups & Oocytes & MII (\%) & 2-cell (\%) & Blastocyst (\%) & Blastocysts cells \\
\hline Control & 770 & $503(64.06 \pm 2.87)^{\mathrm{b}}$ & $262(59.96 \pm 4.54)$ & $43(9.80 \pm 1.93)^{\mathrm{b}}$ & $48.65 \pm 16.56$ \\
Treat & 771 & $563(72.90 \pm 5.13)^{\mathrm{a}}$ & $284(59.75 \pm 3.40)$ & $63(13.28 \pm 1.79)^{\mathrm{a}}$ & $49.65 \pm 18.66$ \\
\hline
\end{tabular}

a, b Values with different superscripts are significantly different $(\mathrm{P}<0.05)$.

\section{Figures}




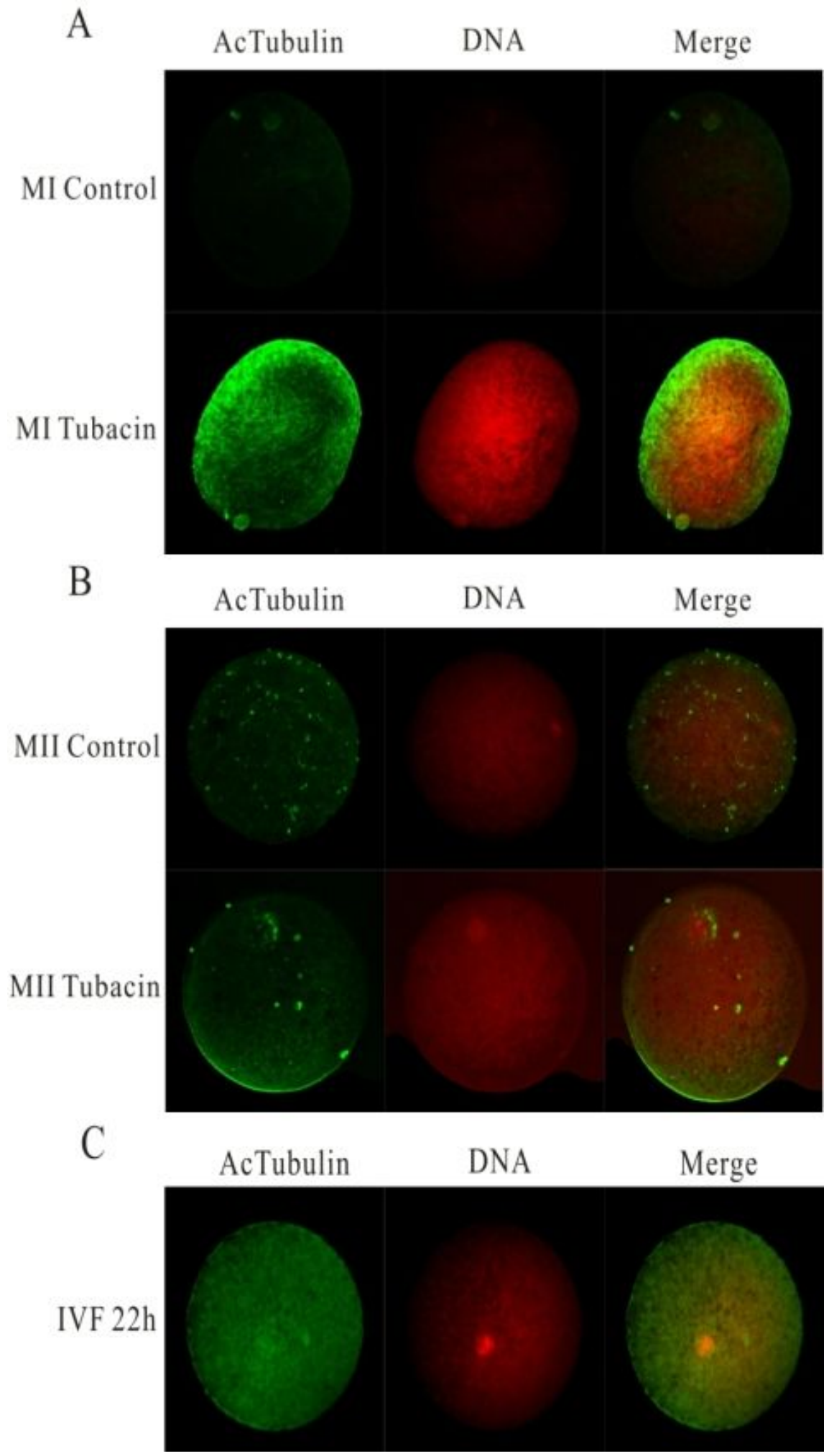

\section{Figure 1}

Comparison of ac-a-Tubulin status of porcine oocytes and embryos during IVM and IVF. Original magnification × 200. Note: A: MI stage; B: MIl stage; C: Syngamy; Green: Ac-a-Tubulin; Red: DNA 


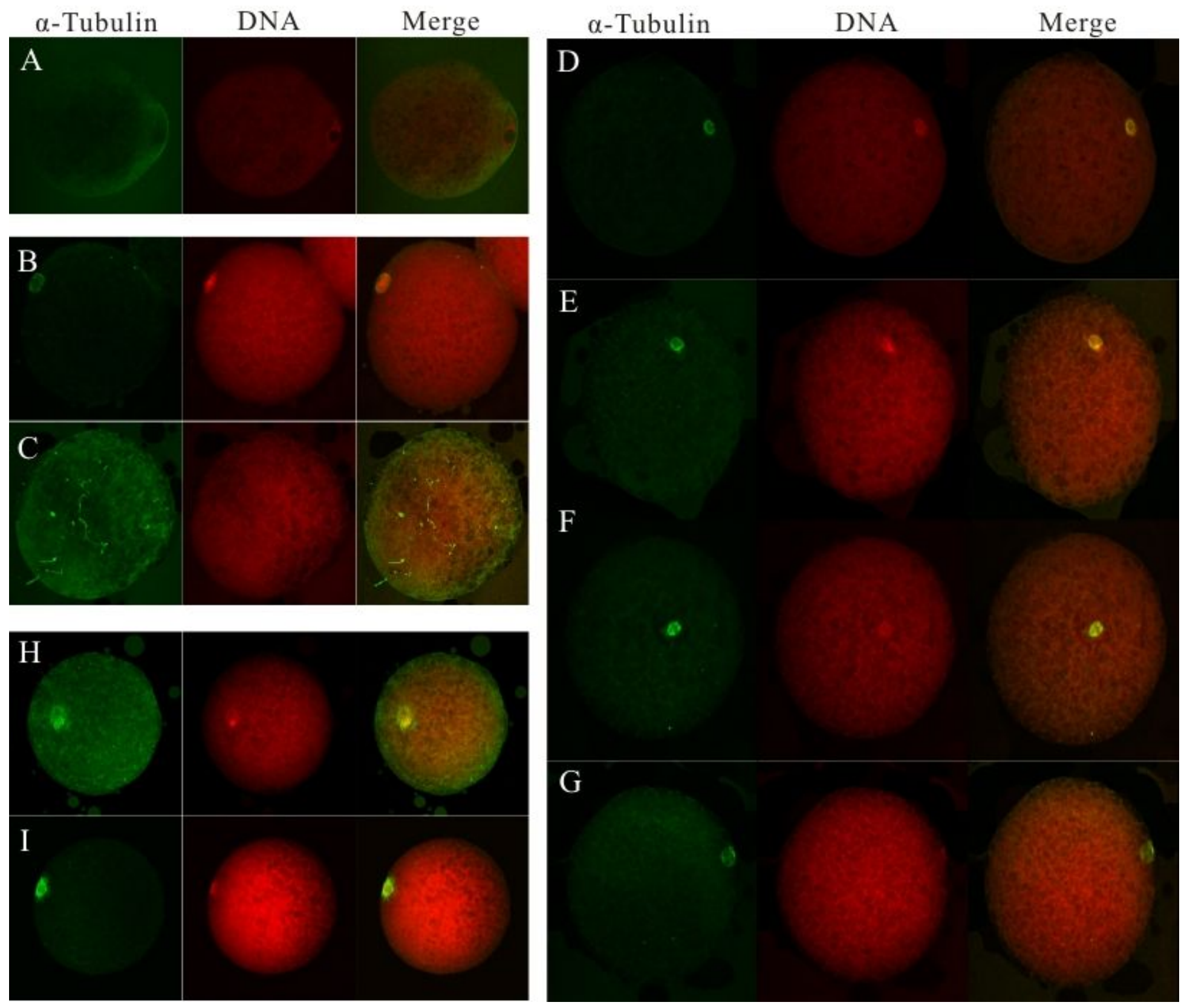

\section{Figure 2}

Comparison of a-tubulin status of porcine oocytes and embryos during IVM and IVF. Original magnification $\times 200$. Note: A: Dispersive distribution of GV oocytes; B: Normal status of Ml oocytes; C: Abnormal status of Ml oocytes; D, E: Normal status of MIl oocytes; F, G: Abnormal status of MIl oocytes; H: Syngamy; I: Unfertilized MII embryo; Green: a-tubulin; Red: DNA. 


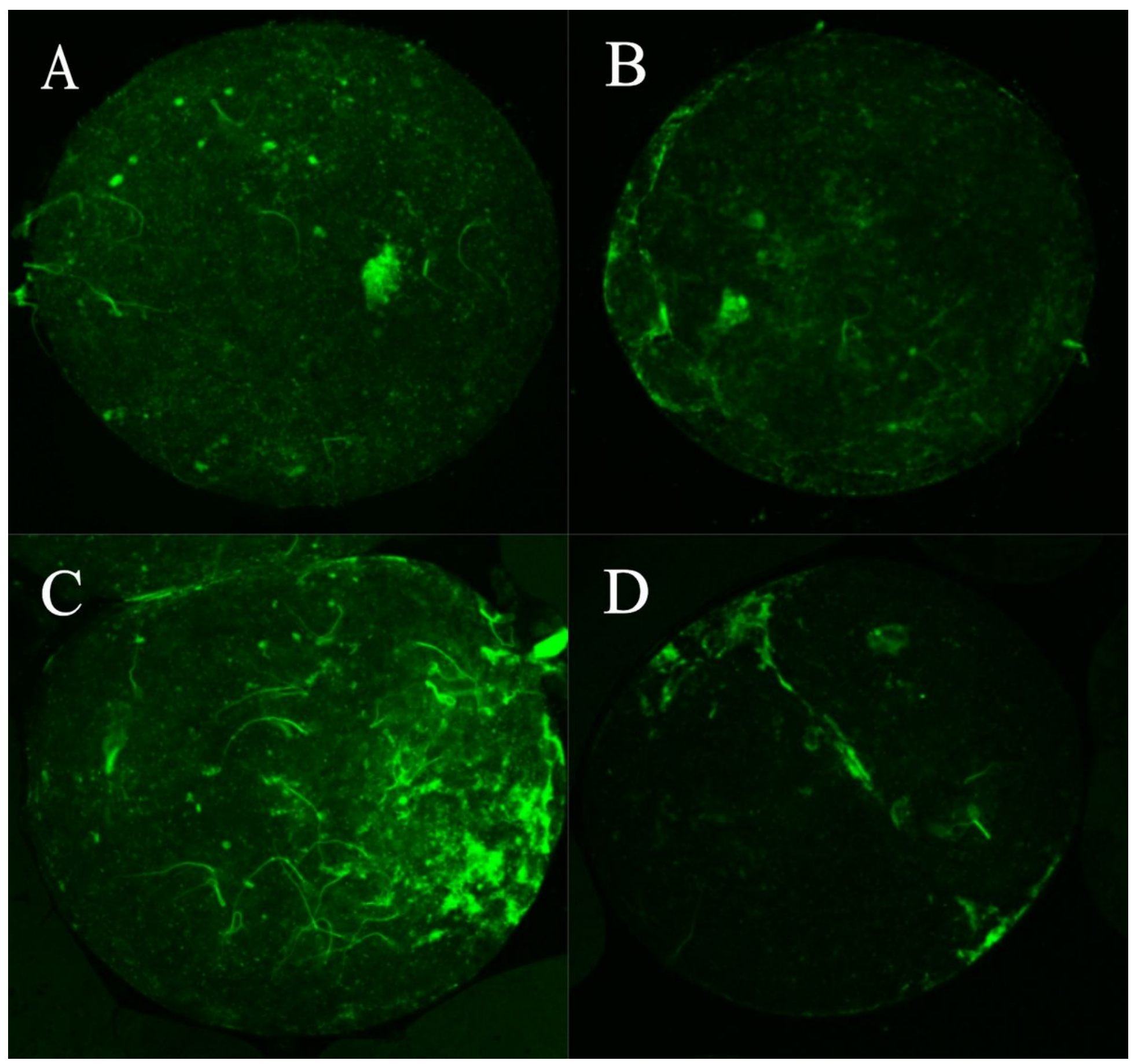

\section{Figure 3}

The mitochondrial distribution in porcine oocytes and embryos. Original magnification $\times 200$. Note: A: Unfertilized MII oocytes; B: Pronuclear stage; C: Syngamy; D: Two-cells stages; Green: mitochondria. 


\section{Pronucleus}

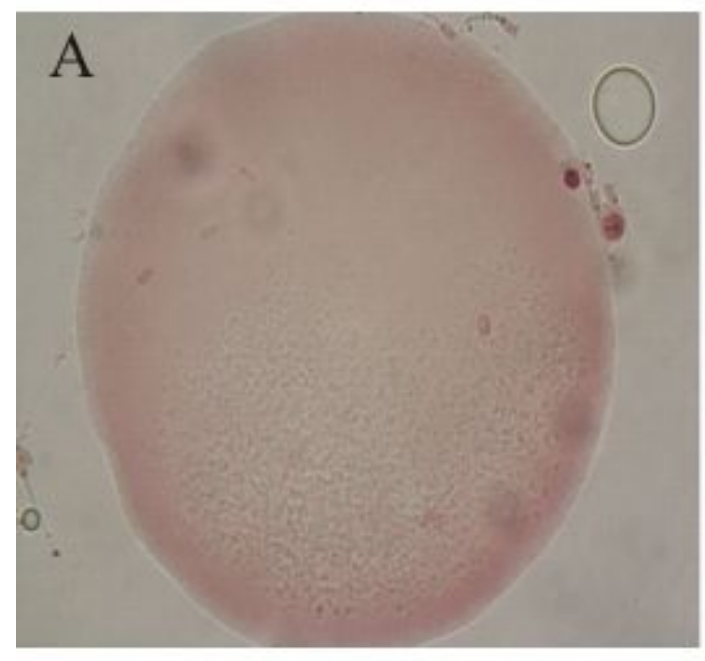

\section{MII}

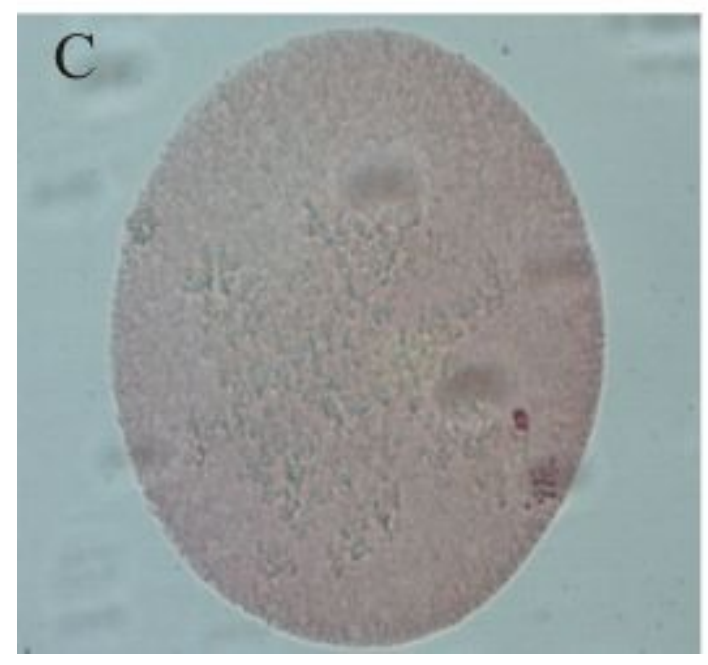

Syngamy

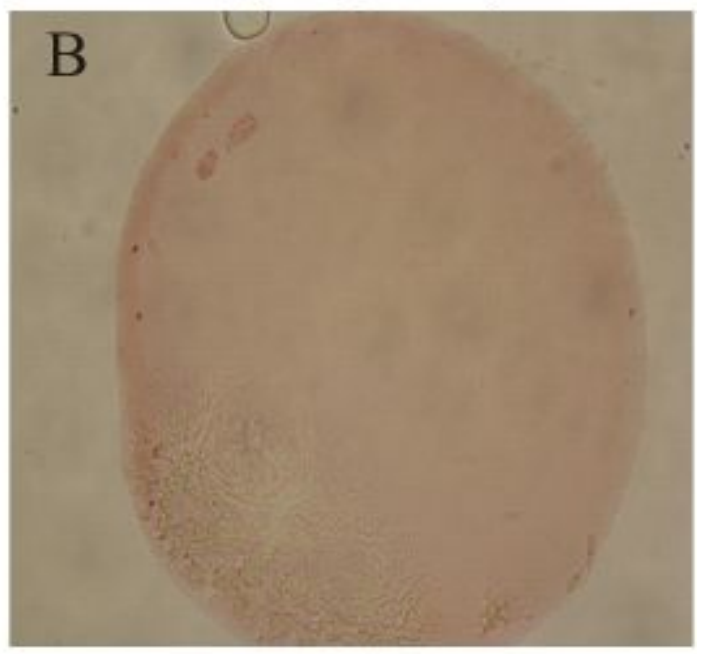

\section{Polysperm}

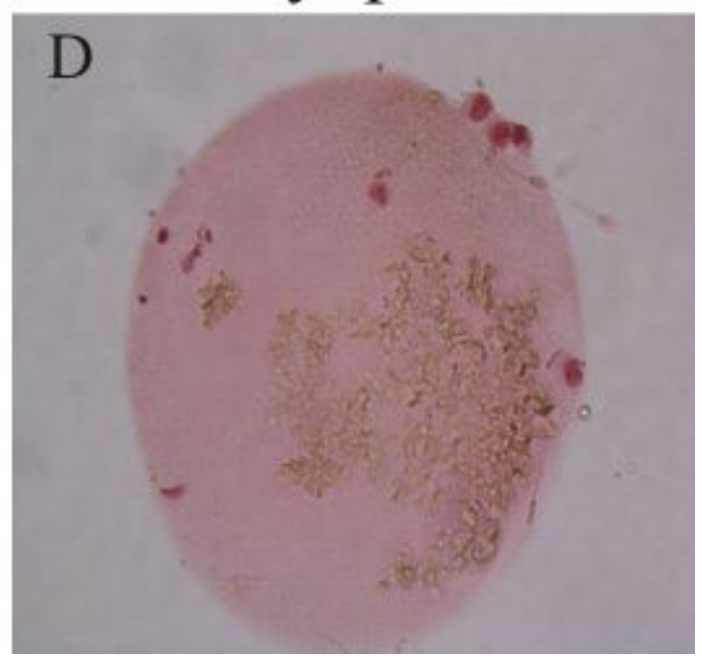

Figure 4

Different nuclear stages of porcine embryos after in vitro fertilization. Original magnification $\times 200$. Note: A: Pronucleus; B: Syngamy; C: Unfertilized; D: Polysperm 

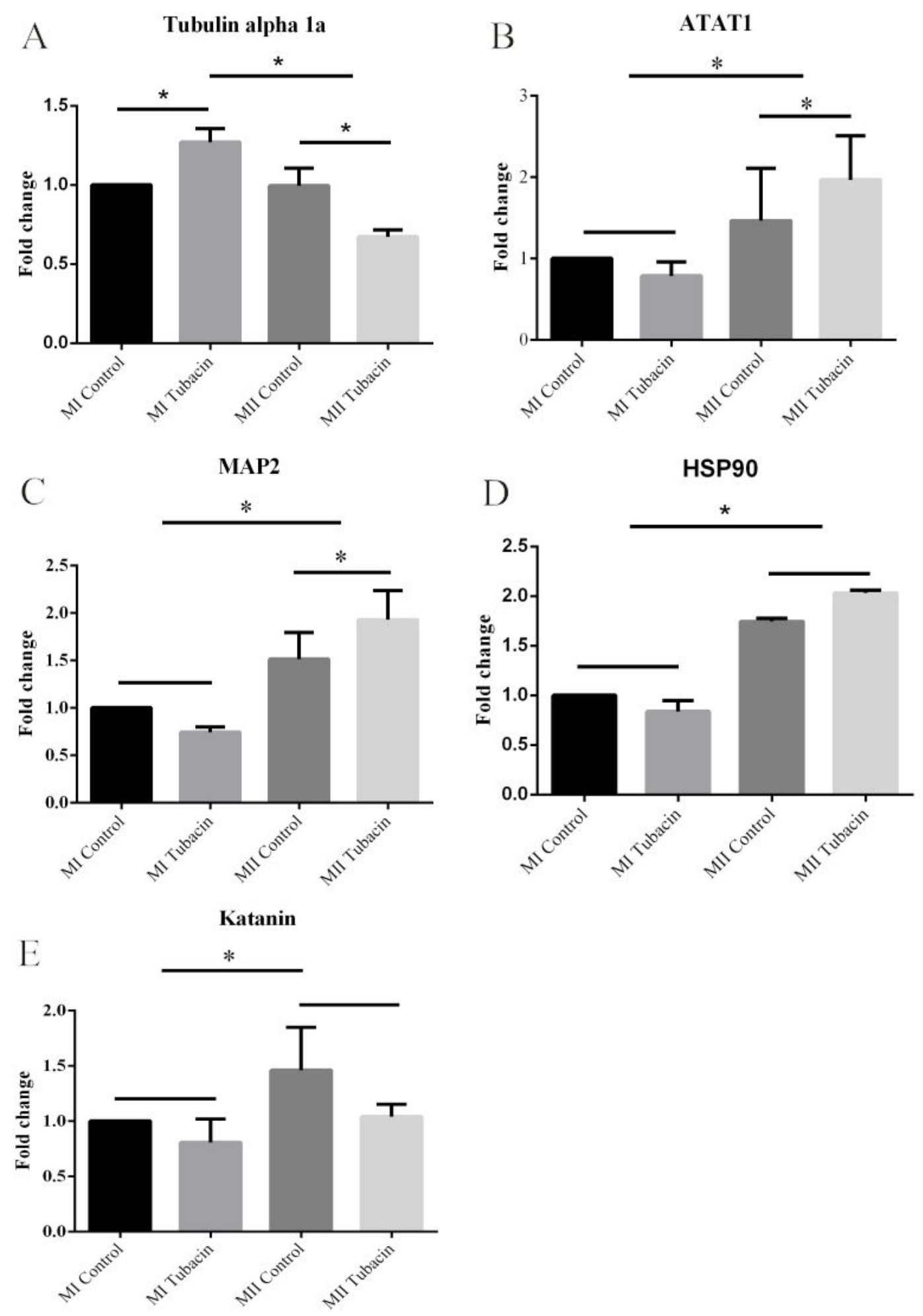

Figure 5

Comparison of relative expression levels of microtubule assembly genes. Note: * Asterisk was significantly different $(P<0.05)$. 